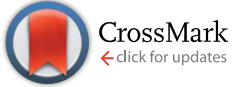

Cite this: Chem. Sci., 2017, 8, 1225

Received 23rd July 2016

Accepted 30th September 2016

DOI: $10.1039 / c 6 s c 03275 c$

www.rsc.org/chemicalscience

\title{
Polymorphism at 129 dictates metastable conformations of the human prion protein $\mathrm{N}$ - terminal $\beta$-sheet $\dagger$
}

\begin{abstract}
S. Alexis Paz, ${ }^{a}$ Eric Vanden-Eijnden ${ }^{\mathrm{b}}$ and Cameron F. Abrams ${ }^{\star a}$
We study the thermodynamic stability of the native state of the human prion protein using a new freeenergy method, replica-exchange on-the-fly parameterization. This method is designed to overcome hidden-variable sampling limitations to yield nearly error-free free-energy profiles along a conformational coordinate. We confirm that all four (M129V, D178N) polymorphs have a ground-state conformation with three intact $\beta$-sheet hydrogen bonds. Additionally, they are observed to have distinct metastabilities determined by the side-chain at position 129. We rationalize these findings with reference to the prion "strain" hypothesis, which links the variety of transmissible spongiform encephalopathy phenotypes to conformationally distinct infectious prion forms and classifies distinct phenotypes of sporadic Creutzfeldt-Jakob disease based solely on the 129 polymorphism. Because such metastable structures are not easily observed in structural experiments, our approach could potentially provide new insights into the conformational origins of prion diseases and other pathologies arising from protein misfolding and aggregation.
\end{abstract}

\section{Introduction}

Transmissible spongiform encephalopathies (TSE's) are a collection of neurodegenerative diseases associated with the misfolding and aggregation of the prion protein (PrP). It is widely accepted that the key phenomenon in pathogenesis is the isomerization of the mostly $\alpha$-helical native cellular protein $\left(\mathrm{PrP}^{\mathrm{C}}\right)$ into a $\beta$-sheet-rich misfolded conformation termed "scrapie" $\left(\mathrm{PrP}^{\mathrm{Sc}}\right){ }^{1}$ The "protein-only" hypothesis maintains that this transformation is catalyzed only by $\operatorname{PrP}^{\mathrm{Sc}}$, which underlies the transmissible nature of prion diseases. However, there is an unusually large spectrum of TSE phenotypes, even within the same animal species, which is difficult to reconcile with the existence of a single $\operatorname{PrP}^{\mathrm{Sc}}$ state. It has therefore been hypothesized that TSE phenotypic differences arise from different $\mathrm{PrP}^{\mathrm{Sc}}$ strains which are primarily marked by conformational differences. $^{2-9}$

A methionine-valine polymorphism observed in codon 129 of the PRPN gene has a strong influence on susceptibility to human prion diseases. ${ }^{\mathbf{1 0 - 1 2}}$ Clinical aspects and neuropathology of sporadic Creutzfeldt-Jakob disease (sCJD), which represents $\sim 75 \%$ of all prion disease cases, ${ }^{\mathbf{8 , 1 3 , 1 4}}$ significantly vary

${ }^{a}$ Department of Chemical and Biological Engineering, Drexel University, Philadelphia, PA 19104, USA. E-mail: cfa22@drexel.edu

${ }^{b}$ Courant Institute of Mathematical Sciences, New York University, New York, NY 10012, USA

$\dagger$ Electronic supplementary information (ESI) available. See DOI: $10.1039 / \mathrm{c} 6 \mathrm{sc} 03275 \mathrm{c}$ depending on whether the patient is homozygous for methionine (MM), for valine (VV) or heterozygous (MV). The 129 polymorphism has acquired a central role in attempts to develop early diagnostic ${ }^{15,16}$ and phenotypic classification ${ }^{6,7,9,17}$ of sCJD. This suggests that residue 129 somehow controls which prion strains emerge in SCJD cases. ${ }^{3}$ Moreover, to date all confirmed cases of variant CJD, which, distressingly, is associated with the epidemic of bovine spongiform encephalopathy, ${ }^{18}$ correspond to MM homozygosity at codon $129,,^{9,19}$ and homozygosity also seems to increase susceptibility to iatrogenic CJD. ${ }^{12,20}$ Finally, one of the most striking examples of 129's influence relates to mutation D178N, which causes genetic CJD if residue 129 is valine and Fatal Familial Insomnia (FFI) if residue 129 is methionine. ${ }^{17,21}$

The lack of a structural basis for the apparent correspondence between phenotypic and genotypic variability, especially in relation to residue 129 , is a major complication in understanding the prion strain phenomenon. To begin to provide such structural insight, we focus in the contribution of the small antiparallel $\beta$ strands of $\operatorname{PrP}^{\mathrm{C}}$. This structure is of particular interest for two reasons. First, the $\beta_{1}$ strand begins precisely at residue 129 . Second, there are at least two conformational changes to $\operatorname{PrP}^{\mathrm{C}}$ that could conceivably lead to the $\beta$-sheet-driven aggregation characteristic of TSE's: (i) elongation of the loop anchored by the $\beta_{1}$ and $\beta_{2}$ strands to form a $\beta$-hairpin that could attach to similar hairpins in other protomers, ${ }^{22}$ and (ii) breaking of the two $\beta$ strands to enable either (a) a registry of $\mathrm{H}$-bonds in the $\beta$ strand distinct from that in native $\operatorname{PrP}^{\mathrm{C}}$ or (b) interprotomer $\beta$-sheet assembly and further 
aggregation. ${ }^{23}$ In this " $\mathrm{PrP}^{\mathrm{C}}$-breaking" hypothesis, the small $\operatorname{PrP}^{\mathrm{C}} \beta$-sheet represents an energy barrier for $\operatorname{PrP}^{\mathrm{C}}$ misfolding and aggregation.

This work is based on the idea that conformational differences among difficult-to-observe metastable intermediates on the $\operatorname{PrP}^{\mathrm{C}} \rightarrow \operatorname{PrP}^{\mathrm{Sc}}$ pathway could underlie 129's multifaceted influence. We explore this idea by direct measurement of the thermodynamics associated with the $\operatorname{PrP}^{\mathrm{C}} \beta$-sheet in all-atom explicit-solvent molecular dynamics (MD) simulations. In particular, we compute the free energy along a collective variable (CV) that measures the degree of "intactness" of the set of four hydrogen bonds that define the $\operatorname{PrP}^{\mathrm{C}} \beta$ sheet. ${ }^{24,25}$ Like all CV's, this one is chosen to represent a process that would never spontaneously occur on limited MD timescales at any physically relevant temperature, so a direct CV-biasing method is required. Importantly, however, we show that thermodynamically comparing the mutants associated with the $129 / 178$ polymorphs requires much higher precision $\left(\ll 1 \mathrm{kcal} \mathrm{mol}^{-1}\right)$ than is typically available in existing free-energy methods, due to the common problem of "hidden variables"; ${ }^{26,27}$ i.e., variables not explicitly biased in the calculation are not ergodically sampled and therefore stymie sampling of the $\mathrm{CV}$ of interest. To meet this challenge in the study of $\operatorname{PrP}^{\mathrm{C}}$, we report here the development of a new free-energy method termed replica-exchange on-the-fly free-energy parameterization (RE-OTFP), which seems uniquely effective at overcoming the hidden-variable problem and is able to produce free-energy profiles of highenough precision to distinguish roles played by point mutations in the stability of the $\operatorname{PrP}^{\mathrm{C}} \beta$-sheet.

This paper is organized as follows. We first present RE-OTFP in context of current methods of CV-biasing in order to explain its advantages. As calibration, we first report its performance on measuring the free energy of the $\beta$ sheet in the mouse $\operatorname{PrP}^{\mathrm{C}}$ relative to other methods. We then describe the detailed simulation protocols used to simulate the four 129/178 doublemutants of human $\operatorname{PrP}^{\mathrm{C}}\left(\mathrm{HuPrP}^{\mathrm{C}}\right)$. We then present and discuss our results, with particular emphasis on the role of 129 in the determination of whether a $\beta$-sheet breaking or elongation conformational phenotype is preferred.

\section{Methods}

\subsection{Replica exchange on-the-fly parameterization}

CV's are a standard way to simplify the description of complex systems. Free-energy minima in CV space correspond to metastable states, and working in CV space therefore provides a framework for identifying such states for further characterization. Projecting the $3 \mathrm{~N}$-dimensional configuration space of the N-atom system $\boldsymbol{x} \in \mathbb{R}^{3 \mathrm{~N}}$ onto a chosen lower-dimensional CV space $z \in \mathbb{R}^{\mathrm{M}}$ via mapping functions $\boldsymbol{\theta}: \boldsymbol{x} \mapsto \mathbb{R}^{\mathrm{M}}$ gives rise to a free-energy landscape (FEL) given by

$$
F(\boldsymbol{z})=-\beta^{-1} \ln \int \delta(\boldsymbol{\theta}(\boldsymbol{x})-\boldsymbol{z}) \mathrm{e}^{-\beta U(\boldsymbol{x})} \mathrm{d} \boldsymbol{x}
$$

where $U(\boldsymbol{x})$ is the potential energy and $\beta=\left(k_{\mathrm{B}} T\right)^{-1}$. In general, the computation of the FEL can be extremely expensive and many methods have been devised over the years to implement such a calculation. ${ }^{28} \mathrm{CV}$ biasing approaches are drastically limited in accuracy and precision due to the common presence of potential-energy barriers on variables not included in the CV space. A major class of methods meant to overcome this hidden-barriers problem are the so-called "orthogonal-space sampling" approaches. ${ }^{29-31}$ However, these methods can only overcome barriers in hidden variables that are strongly coupled with the dynamic of the CVs, and further assumptions on the system are required. ${ }^{32}$ Another approach has been to harness the non-CV-based enhanced sampling approach of parallel

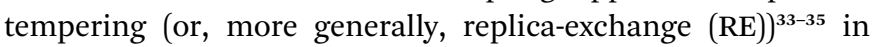
combination with a CV-based method, such as well-tempered metadynamics (WTMD) $)^{\mathbf{2 4 , 3 6}}$ to generate the composite method "PT-WTMD", in which the enhanced sampling of variables other than the CV diminishes the likelihood of hidden-variablebased sampling restrictions. ${ }^{37,38}$ However, popular "adaptive" methods like WTMD and the adaptive-biasing force method ${ }^{\mathbf{3 9 , 4 0}}$ are particularly sensitive to hidden variables because their adaptivity makes them "remember" sampling limitations these variables cause. ${ }^{25}$ This makes non-adaptive methods, such as on-the-fly free-energy parameterization (OTFP) $)^{25,41}$ based on temperature-accelerated MD (TAMD) ${ }^{\mathbf{4 2 , 4 3}}$ preferable. Here we make a brief presentation of OTFP/TAMD.

TAMD introduces auxiliary variables $z$ into an MD simulation, and these are tethered to $\boldsymbol{\theta}(\boldsymbol{x})$ via harmonic springs, such that $\delta(\boldsymbol{\theta}(\boldsymbol{x})-\boldsymbol{z}) \mathrm{e}^{-\beta U(\boldsymbol{x})} \approx \mathrm{e}^{-\frac{\beta k}{2}[\boldsymbol{\theta}(\boldsymbol{x})-\boldsymbol{z}]^{2}} \mathrm{e}^{-\beta U(\boldsymbol{x})} \equiv \mathrm{e}^{-\beta U_{k}(\boldsymbol{x}, z)}$. Replacing the delta-function in eqn (1) with the Gaussian factor defines the mollified estimate of the free energy:

$$
F_{\mathrm{k}}(\boldsymbol{z})=-\beta^{-1} \ln \int \mathrm{e}^{-\beta U_{\mathrm{k}}(\boldsymbol{x}, \boldsymbol{z})} \mathrm{d} \boldsymbol{x} .
$$

The equations of motion of $z$ are tuned such they feel negative gradients in $F_{\mathrm{k}}(z)$ as if they were conservative forces; providing the $z$ with a higher "auxiliary" temperature $\bar{\beta}^{-1}>\beta^{-1}$ gives them the ability to overcome free-energy barriers and therefore better sample CV space than would a projection of any finite thermal MD simulation. OTFP accumulates the estimators of $\nabla F(z)$ in a TAMD simulation and, positing a basis-function expansion of $F(z)$, uses them to find optimal coefficients in that expansion, providing an estimate for $F(z)$. That is, TAMD ensures good sampling of CV space by overcoming free-energy barriers and OTFP actually computes the FEL. ${ }^{\mathbf{4 1}} \mathrm{A}$ key feature in the efficiency of OTFP is that estimates of local free-energy gradients converge more quickly than does the uniformity of sampling of CV-space.

Replica-exchange molecular dynamics (REMD) can be implemented on top of TAMD in the following way. Consider an artificial system built up of $R$ replicas of a TAMD system, each replica with its own real and auxiliary temperatures. Replicas are labeled following the convention $\beta_{1}>\beta_{2}>\ldots>\beta_{R}$ and the auxiliary temperatures can be taken arbitrarily. Let $\rho\left(\boldsymbol{x}_{1}, z_{1}, \ldots, \boldsymbol{x}_{R}, z_{R}\right)$ denote the joint distribution of the $R$ replicas. It is given by

$$
\rho\left(\boldsymbol{x}_{1}, \boldsymbol{z}_{1}, \ldots, \boldsymbol{x}_{R}, \boldsymbol{z}_{R}\right)=\frac{1}{R !} \sum_{\sigma} \prod_{i=1}^{R} \rho\left(\boldsymbol{x}_{i}, \boldsymbol{z}_{i}, \boldsymbol{\beta}_{\sigma(i)}, \overline{\boldsymbol{\beta}}_{\sigma(i)}\right)
$$


here $\sigma$ denotes permutation over the $R$ indices, and we defined

$$
\rho(\boldsymbol{x}, \boldsymbol{z}, \beta, \bar{\beta})=\rho(\boldsymbol{x} \mid \boldsymbol{z}, \beta) \rho(\boldsymbol{z}, \beta, \bar{\beta})
$$

where

$$
\rho(\boldsymbol{x} \mid \boldsymbol{z}, \beta, \bar{\beta})=\frac{\mathrm{e}^{-\beta U_{\mathrm{k}}(\boldsymbol{x}, z)}}{\int \mathrm{e}^{-\beta U_{\mathrm{k}}(\boldsymbol{x}, \boldsymbol{z})} \mathrm{d} \boldsymbol{x}} \equiv \frac{\mathrm{e}^{-\beta U_{\mathrm{k}}(\boldsymbol{x}, z)}}{\mathrm{e}^{-\beta F_{\mathrm{k}}(\boldsymbol{z}, \beta)}},
$$

and

$$
\rho(\boldsymbol{z}, \beta, \bar{\beta})=\bar{Z}^{-1}(\beta, \bar{\beta}) \mathrm{e}^{-\bar{\beta} F_{\mathrm{k}}(z, \beta)}, \quad \bar{Z}(\beta, \bar{\beta})=\int \mathrm{e}^{-\bar{\beta} F_{\mathrm{k}}(z, \beta)} \mathrm{d} \boldsymbol{z}
$$

Eqn (4) says that the $\boldsymbol{x}$ variables are effectively at equilibrium with (i.e., adiabatically slaved to) the $z$ variables and feel the temperature $\beta$, whereas the variables $z$ navigate on the mollified free energy computed at temperature $\beta, F_{\mathrm{k}}(z, \beta)$, but feel the auxiliary temperature $\bar{\beta}$. Assume now that we attempt to exchange the temperatures between replica $i$ having positions $\left(\boldsymbol{x}_{i}, z_{i}\right)$ and temperatures $\left(\beta_{i}, \bar{\beta}_{i}\right)$, and replica $j$ having positions $\left(\boldsymbol{x}_{j}\right.$, $z_{j}$ ) and temperatures $\left(\beta_{j}, \bar{\beta}_{j}\right)$, so that, if the move is accepted, immediately after this move replica $i$ is now at temperatures $\left(\beta_{j}, \bar{\beta}_{j}\right)$ and replica $j$ at temperatures $\left(\beta_{i}, \bar{\beta}_{i}\right)$, with their respective positions unchanged. The metropolis acceptance probability of this move is

$$
A_{i \leftrightarrow j}=\min \left\{1, \frac{\rho\left(\boldsymbol{x}_{i}, \boldsymbol{z}_{i}, \beta_{j}, \bar{\beta}_{j}\right) \rho\left(\boldsymbol{x}_{j}, \boldsymbol{z}_{j}, \beta_{i}, \bar{\beta}_{i}\right)}{\rho\left(\boldsymbol{x}_{i}, \boldsymbol{z}_{i}, \beta_{i}, \bar{\beta}_{i}\right) \rho\left(\boldsymbol{x}_{j}, \boldsymbol{z}_{j}, \beta_{j}, \bar{\beta}_{j}\right)}\right\}
$$

Using (4), the factor involving ratios of $\rho$ 's can be written explicitly

$$
A_{i \leftrightarrow j}=\min \left\{1, \exp \left(\Delta_{i, j}\right)\right\}
$$

where

$$
\begin{aligned}
\Delta_{i, j}=\left(\beta_{j}-\beta_{i}\right)\left(U_{\mathrm{k}}\left(\boldsymbol{x}_{j}, \boldsymbol{z}_{j}\right)-U_{\mathrm{k}}\left(\boldsymbol{x}_{i}, \boldsymbol{z}_{i}\right)\right)+\left(\bar{\beta}_{j}-\beta_{j}\right)\left(F_{\mathrm{k}}\left(\boldsymbol{z}_{j}, \beta_{j}\right)\right. \\
\left.-F_{\mathrm{k}}\left(\boldsymbol{z}_{i}, \beta_{j}\right)\right)+\left(\left(\bar{\beta}_{i}-\beta_{i}\right)\left(F_{\mathrm{k}}\left(z_{i}, \beta_{i}\right)-F_{\mathrm{k}}\left(\boldsymbol{z}_{j}, \beta_{i}\right)\right)\right.
\end{aligned}
$$

As can be appreciated from eqn (8), the acceptance criterion requires as input the free energies at the two replica temperatures, $F\left(z, \beta_{i}\right)$ and $F\left(z, \beta_{j}\right)$, each evaluated at both points in $z$-space, $z_{j}$ and $z_{i}$. We propose here to use OTFP to estimate the free energy differences needed in the acceptance criterion. As the simulation progresses, the computed FEL of each replica will be continuously refined via OTFP.

Exchange moves between adjacent replicas are attempted at regular intervals. Furthermore, many trials can be performed simultaneously: it is a common practice to consider only neighboring replicas for exchange which allows $\sim R / 2$ independent simultaneous attempts. In each attempt, the (groups of) processors running the $i$ th and $j$ th replica communicate to each other their instantaneous values of $z_{i}$ and $z_{j}$ respectively. Then, each processor can compute the values of $F\left(z_{j}, \beta_{i}\right)-F\left(z_{i}, \beta_{i}\right)$ and $F\left(z_{j}, \beta_{j}\right)-F\left(z_{i}, \beta_{j}\right)$ as needed. One of the processors communicates back the value obtained and its instantaneous value of $U_{\mathrm{k}}(\boldsymbol{x}, \boldsymbol{z})$. The other processor will use this information to compute if the exchange is accepted using eqn (8). If is accepted, only the real and auxiliary temperatures are swapped. Finally, the momenta associated with $\boldsymbol{x}_{i}$ and $\boldsymbol{x}_{j}$ are rescaled using the factors $\sqrt{\beta_{j} / \beta_{i}}$ and $\sqrt{\beta_{i} / \beta_{j}}$ respectively. ${ }^{44}$ Since we evolve the auxiliary variables using Brownian dynamics, there is no momentum associated with them. However, if different stochastic dynamics are used, equivalent scaling factors using the auxiliary temperatures should be applied.

We have given the name "replica-exchange on-the-fly freeenergy parameterization" (RE-OTFP) to the present combination of REMD and TAMD with OTFP. Note that the RE-OTFP has similarities with PT-WTMD. ${ }^{37}$ This becomes evident in the comparison of the acceptance rules for both methods: it is possible to shown that the two terms with the free-energy differences in eqn (9) are equivalent to the two bias terms in the acceptance rule of PT-WTMD. The analogy between RE-OTFP and PT-WTMD is not surprising, considering the similarities between OTFP and WTMD pointed out in our previous work. ${ }^{25}$ Note, however, that there is no history-dependent bias in RE-OTFP.

\subsection{Simulation set-up and protocols}

The number of replicas and their corresponding temperatures are important choices that can drastically affect sampling in a replica exchange simulation. Following Kone and Kofke, ${ }^{45}$ the average acceptance probability can be approximated as

$$
\left\langle A_{i \leftrightarrow j}\right\rangle=\operatorname{erfc}\left(\frac{1-T_{i} / T_{j}}{1+T_{i} / T_{j}} \sqrt{C}\right)
$$

where $C$ is the (extensive) heat capacity (in units of the Boltzmann constant), assumed to be constant in the temperature range between the involved replicas. We have computed $C$ of the mouse $\operatorname{PrP}^{\mathrm{C}}$ at different temperatures using 10 molecular dynamic simulations (see ESI $\dagger$ ). The average heat capacity obtained is $61 \mathrm{kcal}(\mathrm{mol} \mathrm{K})^{-1}$. For the sake of simplicity we take this value as constant for the temperature range studied. With this assumption, we can use eqn (10) to write

$$
\frac{T_{j}}{T_{i}}=\left(\frac{\sqrt{C}+\operatorname{erfc}^{-1}\left(A_{\leftrightarrow}\right)}{\sqrt{C}-\operatorname{erfc}^{-1}\left(A_{\leftrightarrow}\right)}\right)^{(j-i)}
$$

where $A_{\leftrightarrow}$ is the average acceptance probability between neighboring replicas. It has been suggested that a value near 0.2 might constitute an optimal set up for this quantity. ${ }^{45,46}$ By fixing $T_{2}=300 \mathrm{~K}$ and $T_{N}=400 \mathrm{~K}$ we use eqn (11) to predict that a number $N=30$ replicas is necessary to obtain $A_{\leftrightarrow}=0.2$. Replica temperatures were fixed using the same equation.

Another fundamental factor in the success of an RE-OTFP simulation is the choice of the auxiliary temperatures for each replica. This parameter defines the acceleration strength of each TAMD replica and will also affect the acceptance probability of the exchange between them (eqn (8)). In the present study we choose a constant auxiliary temperature $\bar{T}_{i}=3000 \mathrm{~K}$ for all replicas. This is the same auxiliary temperature using in our previous work, ${ }^{25}$ which means that the replica here at $300 \mathrm{~K}$ will experience the same bias as in that work. However, other choices for $\bar{T}_{i}$ are possible and will be carefully studied in future works. 
All simulations presented in this work were performed using NAMD 2.9 (ref. 47) and the CHARMM force field ${ }^{48}$ with CMAP corrections. ${ }^{49}$ The system details as well as the equilibration procedure used to obtain the initial configuration of the mouse prion protein (MoPrP) are described elsewhere. ${ }^{25}$ An analogous protocol was used for the human prion protein HuPrP-129M: after solvating the NMR structure ${ }^{50}$ (PDB ID: $1 \mathrm{HJM}$ ), $110 \mathrm{~ns}$ of equilibration were run while keeping the protein fixed during the first 5 ns. Mutant D178N-129M was constructed by substituting residue 178 in this equilibrated system and was subsequently equilibrated for $50 \mathrm{~ns}$. The 129-valine polymorphs were constructed substituting residue 129 in the HuPrP-129M and D178N$129 \mathrm{M}$ equilibrated structures and were subsequently equilibrated another $50 \mathrm{~ns}$. The details of these preliminary simulations and their RMSDs are included in the ESI. $\dagger$

RE-OTFP replicas were simulated in the NVT ensemble in agreement with eqn (8). The temperature was controlled using a Langevin thermostat with a damping constant of $50 \mathrm{ps}^{-1}$. We devised a standard protocol for RE-OTFP simulations comprising four stages:

Initialization. The FEL is considered unknown and therefore no exchanges are allowed. This stage is used to build the first FEL guess using OTFP.

Equilibration. This simulation stage is used to allow the different replicas to decorrelate from the initial state. Exchanges are allowed and the FEL is constantly refined.

Reset. At the beginning of this stage the statistics accumulated during the previous stages are forgotten. This is intended to prevent any correlation with non-ergodic sampling that may have arisen in earlier stages. The FEL refinement process starts again but in the background while the replica exchanges are performed using the FEL obtained at the end of the equilibration.

Production. The FEL obtained in the reset stage is used to perform the replica exchanges and is continuously refined onthe-fly until the end of the simulation.

All RE-OTFP simulations of the prion protein genotypes use $1 \mathrm{~ns}$ of initialization, $8 \mathrm{~ns}$ of equilibration and $1 \mathrm{~ns}$ in the reset stage. The production stage involve $18 \mathrm{~ns}$ for MoPrP system and 28 ns for HuPrP and D178N polymorphs.

\subsection{Validating RE-OTFP with alanine dipeptide}

As a further validation of the present RE-OTFP method, we include in Section 3 of the ESI $\dagger$ the 1-D FEL reconstructions for the $\psi$ and $\phi$ angles of alanine dipeptide in water. The simulation parameters for this system where identical to those used for the prion systems. The obtained profiles were compared with those obtained from $74 \mathrm{~ns}$ of pure REMD and showed excellent agreement.

\section{Results}

\subsection{RE-OTFP performance on mouse $\operatorname{Prp}^{\mathrm{C}}$}

We first characterize the performance of RE-OTFP by reconstructing the FEL of the $\beta$-sheet structure of the murine prion protein. The $\mathrm{CV}$ is

$$
s_{4}(\boldsymbol{x})=\sum_{i=1}^{4} \frac{1}{1+\left(r_{i}(\boldsymbol{x}) / 2.5\right)^{6}},
$$

where $r_{i}$ is the $\mathrm{H}-\mathrm{N}$ distance of $\mathrm{H}$-bond $i$ out of the set of four $\mathrm{H}$ bonds formed between the two short $\beta$-strands 129-131 and 161-163. Fig. 1 indicates $\mathrm{H}$-bond 1 to $\mathrm{H}$-bond 4 as well as $\mathrm{H}$ bond 5 and $\mathrm{H}$-bond 6 which are not included in the CV.

We performed three independent RE-OTFP simulations, each consisting of 30 replicas spanning from $295 \mathrm{~K}$ to $400 \mathrm{~K}$ and lasting $28 \mathrm{~ns}$ (18 ns of production). The FELs obtained were in excellent agreement among the three independent simulations, as shown in Fig. 2a for four representative temperatures. It is remarkable that this agreement persists even for the free-energy shoulder at $s_{4} \approx 3.2$, which we showed previously suffers very large errors using WTMD and non-RE OTFP, due to hidden variables. ${ }^{25}$ In Fig. 2 b we show a comparison between the FEL obtained at $300 \mathrm{~K}$ via RE-OTFP and the corresponding profile obtained via averaging 16 independent OTFP simulations of 60 ns each from our previous work. ${ }^{25}$ The remarkable precision observed for the RE-OTFP profiles indicates that the method is able to overcome the hidden barriers that underlie error in this region of $\mathrm{CV}$ space and thereby achieve high reproducibility. The dispersion of these profiles is small enough to establish that an independent RE-OTFP simulation can adequately represent the average to much less than $1 \mathrm{kcal} \mathrm{mol}^{-1}$. Despite the small overhead due to replica exchange, the computational effort for a single RE-OTFP simulation yielding sub-kcal errors is $840 \mathrm{~ns}(30 \times 28)$, which is somewhat lower than the $960 \mathrm{~ns}(16$ $\times 60$ ) used to obtain the FEL via OTFP with errors of greater than $4 \mathrm{kcal} \mathrm{mol}{ }^{-1} \cdot{ }^{25}$ We show in the $\mathrm{ESI}^{\dagger}$ (Sections 3 and 5) evidence that RE-OTFP outperforms OTFP and REMD separately in both sampling the $\mathrm{CV}$ and overcoming hidden-variable sampling issues.

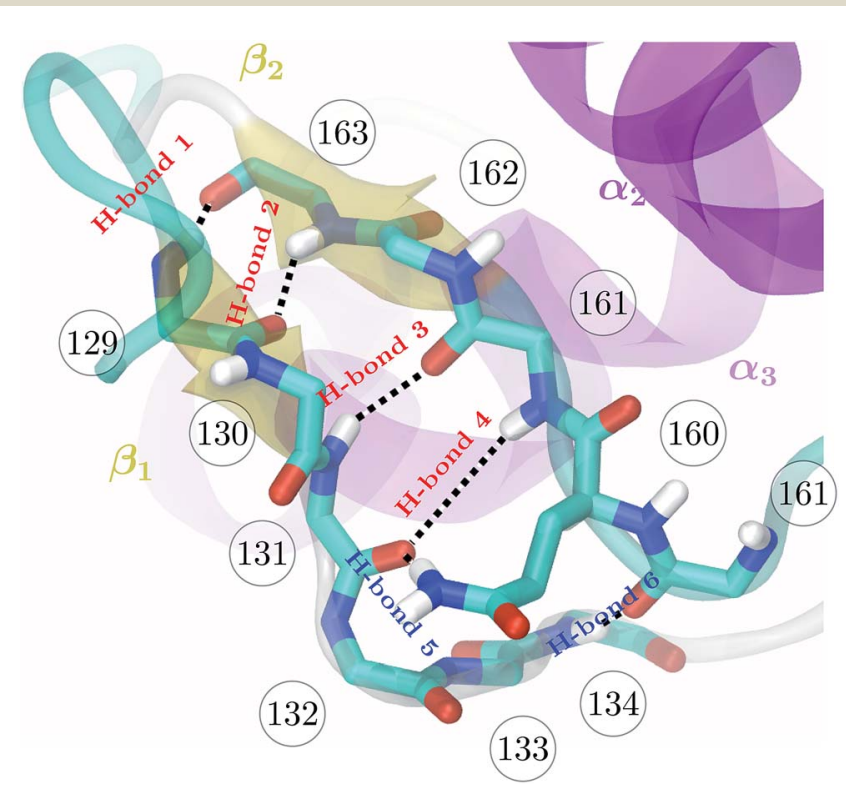

Fig. 1 Backbone of the $\beta$-sheet structure of the mouse prion protein. Secondary structure, residue numbers and $\mathrm{H}$-bonds labels are included. 

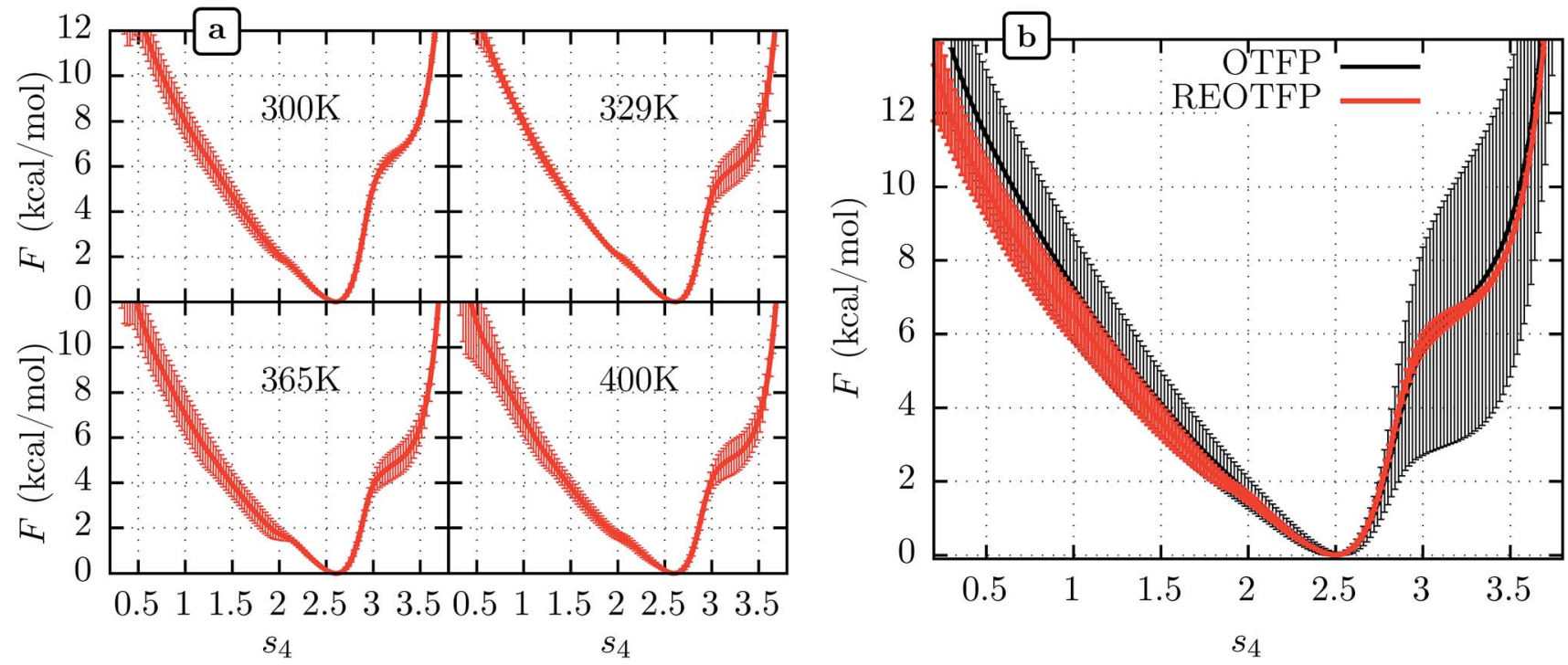

Fig. 2 (a) Average free energy landscape (FEL) along $s_{4}$ for the MoPrPC at different temperatures obtained via RE-OTFP simulations. (b) Comparison between FELs obtained via RE-OTFP and pure OTFP. ${ }^{25}$ Error bars correspond to \pm one standard deviation from independent simulations (RE-OTFP, $n=3$; OTFP, $n=16$ ).

The higher precision obtained via RE-OTFP relative to OTFP allows observation of finer details of the FEL's. Fig. 2a shows the averaged FEL for $s_{4}$ in the $\mathrm{MoPrP}^{\mathrm{C}}$ at different temperatures. We observe that the free energy shoulder at $s_{4}>3$ decreases with increasing temperature. This effect can be explained on the basis of the competition between H-bond 4 and H-bond 5 . While the acceptor group for H-bond 4 is in the backbone of residue 131, H-bond 5 involves the side chain of residue 160 . When the temperature increases, it is reasonable to expect an increase in the side-chain flexibility and thus $\mathrm{H}$-bond 5 will be weakened, allowing $\mathrm{H}$-bond 4 to form and contribute to $s_{4}$. In the opposite direction, Fig. 2 shows the formation of a smaller shoulder near $s_{4} \approx 1.8$ indicating destabilization of the $\beta$-sheet at high temperatures. By inspection of the corresponding configurations, we can relate this shoulder to the full detachment of $\mathrm{H}$-bond 3 leading only $\mathrm{H}$-bond 1 and 2 remaining intact.

\subsection{Human $\operatorname{PrP}^{\mathrm{C}}$ : effects of 129 and 178 polymorphisms on $\beta$-sheet stability}

We show FEL's at physiological temperature (309 K) for the human $\operatorname{PrP}^{\mathrm{C}}\left(\mathrm{HuPrP}^{\mathrm{C}}\right)$ for both $\mathrm{M}$ and $\mathrm{V}$ genotypes at position 129 in Fig. 3a. We see that in both cases the thermodynamically most stable state falls around $s_{4}=2.5$, similar to MoPrP. However, significant differences appear in the free energy of the surrounding subdomains. While the valine genotype displays a FEL similar to that of $\mathrm{MoPrP}^{\mathrm{C}}$, the methionine genotype shows a striking secondary free-energy minimum at $s_{4} \approx 1.8$. In Fig. $3 \mathrm{~b}$ we show the corresponding FEL's for the D178N mutant. Again, the most stable state in all cases is at $s_{4}=2.5$. With the D178N mutation, however, the 129 polymorphism has the opposite effect on the metastabilities on $s_{4}$ relative to WT: the valine genotype shows a secondary minimum at $s_{4} \approx 1.8$ while the methionine genotype shows no such secondary minimum.
One reasonable interpretation of the secondary minimum at $s_{4} \approx 1.8$ is as an indicator of a propensity of the $\beta$-sheet to unfold. ${ }^{23}$ We see that the valine genotype at 129 therefore stabilizes the $\beta$-sheet in the D178 background but destabilizes it in the N178 background. Within this interpretation, a comment must be added in respect to the minimum observed at $s_{4} \approx 0.8$ in D178N-129M (Fig. 3b). While this minimum indicates another metastable state present at low $s_{4}$, it has a free energy of $4.5 \mathrm{kcal} \mathrm{mol}^{-1}$ above the global minimum, which implies a marginal population of near the $0.3 \%$ of the equilibrium configuration at $s_{4} \approx 2.5$. In contrast, the secondary minima at $s_{4} \approx 1.8$ present in D178N-129V and HuPrP-129M is expected to be more significant in the $\beta$-sheet stability because it represent nearly the $20 \%$ of the equilibrium configuration.

Although the 178 polymorphism has an effect on the $\mathrm{H}$-bond network in the ground-state $\operatorname{PrP}^{\mathrm{C}}$ conformations, an effect of the 129 polymorphism on these states is not apparent. Fig. 4 shows the H-bond network near residue 178 for the equilibrium configurations (i.e. $2.4<s_{4}<2.6$ ) at $309 \mathrm{~K}$ from the REOFTP simulations. The different line widths in the schematic represents the frequency at which we observe each $\mathrm{H}$-bond at a bond distance below $2.5 \AA$ A In the wild-type, the side chain of D178 acts as an H-bond donor and bind the side chain of R164 and Y128. After the mutation D178N is introduced, R164 forms a strong H-bond with E168 and no longer interacts with 178. However, no significant differences in the H-bond network were observed between the different 129 genotypes in the D178N mutant or in the wild-type. The same observations remain true also in the $\mathrm{H}$-bond networks for $s_{4} \approx 1.8$ (see Fig. S11 in the $\mathrm{ESI} \dagger$ ).

The FEL for the wild-type and mutant polymorphs predicts an equilibrium configuration of $s_{4} \approx 2.5$ in all cases. This is in agreement with the crystal structures for D178N-M129, D178NV129 and the three structures for HuPrP-129V reported by Lee 


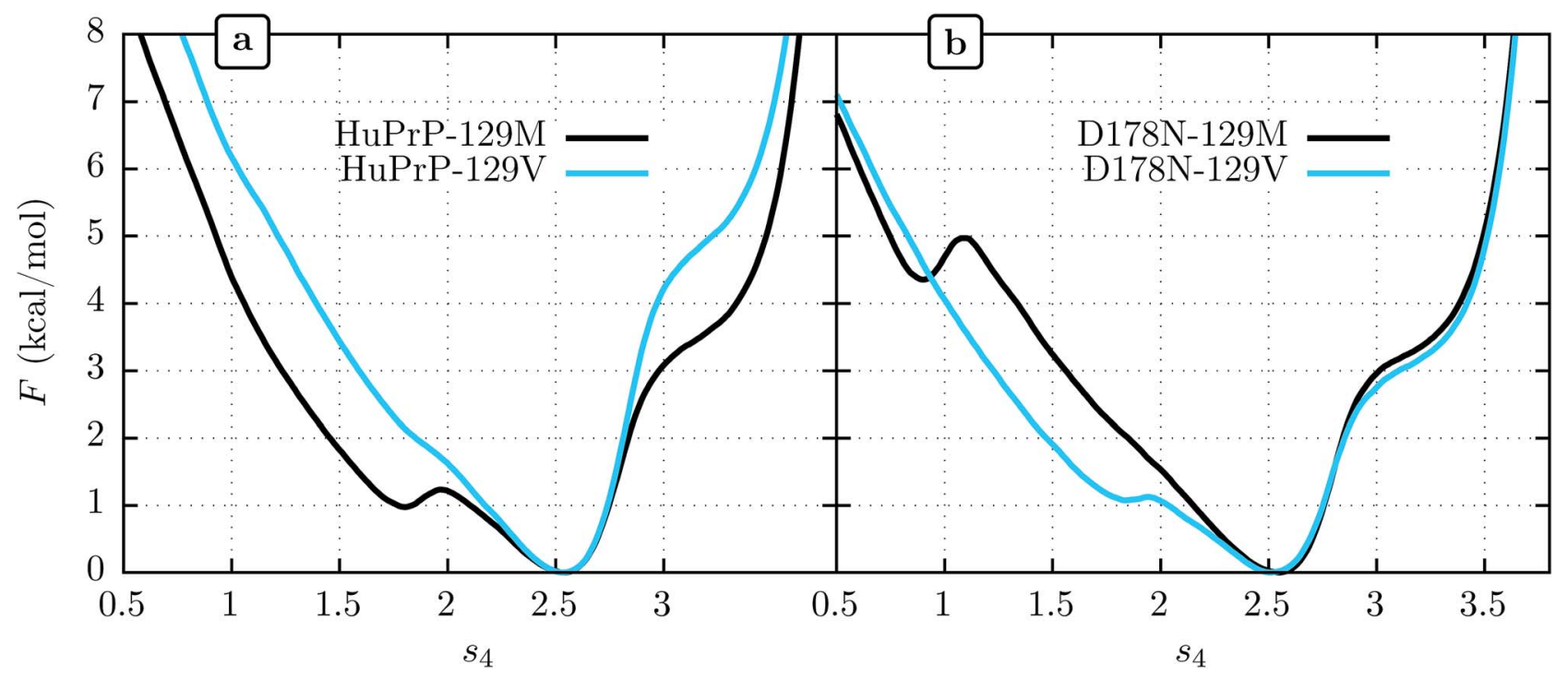

Fig. 3 Free energy vs. $s_{4}$ for human wild-type $\operatorname{PrP}^{C}$ (a) and mutant D178N (b).

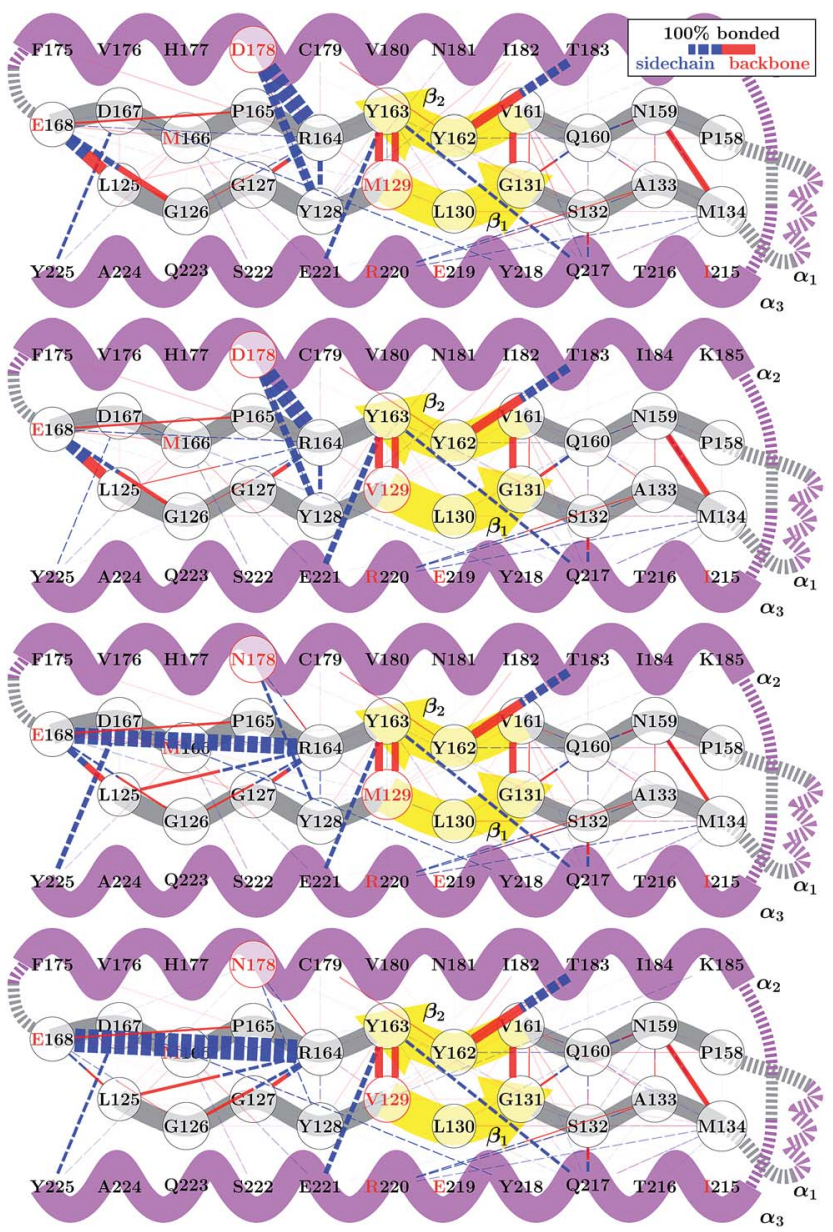

Fig. 4 Schematic of the $\mathrm{H}$-bond network near the $\beta$-sheet structure of WT HuPrPC and the D178N mutant. Bond thicknesses indicate relative frequencies with which $\mathrm{H}-\mathrm{O}$ bond lengths below $2.5 \AA$ are observed at $309 \mathrm{~K}$. et al. ${ }^{51}$ After adding the corresponding hydrogens to these structures we measure values of $s_{4}=2.57,2.52,2.65,2.61$ and 2.51 respectively. Nonetheless, a detailed comparison between the H-bond network from our simulations, presented in Fig. 4, and networks observed in crystal structures is not straightforward. Lee et al. observed several different configurations for the H-bond network surrounding residues 129 and 178, indicating high variability in the side-chain packing for this area. ${ }^{51}$ The present results also resemble those of Hosszu et al. ${ }^{52}$ where the NMR and crystal structure for the two wild type polymorphs are shown to closely match. ${ }^{52}$ Furthermore, Hosszu et al. find no significant differences in local dynamics and structural stability in measurements of amide protection factors and chemical shifts, which is consistent with our present picture of the H-bond network.

\section{Discussion}

It has been proposed that conformational changes in the native $\beta$-sheet structure may have an important role in the $\operatorname{PrP}^{\mathrm{C}} \rightarrow$ $\mathrm{PrP}^{\mathrm{Sc}}$ conversion. The main reason to focus on this small structure is that this conversion should be accompanied by a large increase in the $\beta$-sheet content of $\operatorname{PrP}^{\mathrm{C}}$. However, two seemingly contrary hypotheses arise from considering the role played by this structure: the $\beta$-sheet serves as a seed that guides the elongation as an early stage of the $\operatorname{PrP}^{\mathrm{C}}$ transformation ${ }^{22}$ or the native $\beta$-sheet must break to allow the transformation to occur. ${ }^{23,53}$ The results obtained in the present work motivate us to contribute a different hypothesis to this discussion: crossing an energetic barrier associated first with rupture of the native $\beta$ sheet or first with elongation of the native $\beta$-sheet each commit $\mathrm{PrP}^{\mathrm{C}}$ to distinct infectious $\mathrm{PrP}^{\mathrm{Sc}}$ forms, and the polymorphism at 129 can favor one or the other. The minima observed at $s_{4} \approx$ 1.8 indicate that the methionine genotype seems to be more susceptible to breaking of the native $\beta$-sheet structure (Fig. 3). The FEL for the valine genotype indicates an improved native $\beta$ - 
sheet stability at physiological temperatures, which is consistent with the idea of an elongation of the native $\beta$-sheet structure as a mechanism of conversion.

Modern classification schemes for sporadic CJD phenotypes originated with Parchi et al. ${ }^{6-8}$ In this scheme, two different $\operatorname{PrP}^{\mathrm{Sc}}$ types are defined, and according to patient allele at codon 129, six sporadic CJD sub-types are identified. These sub-types conform very well to the clinical and histological observations. The evidence suggests that each $\mathrm{PrP}^{\mathrm{Sc}}$ type arises from different $\mathrm{PrP}^{\mathrm{Sc}}$ conformations. At the same time, a strong correlation exists between the $\mathrm{PrP}^{\mathrm{Sc}}$ type and the codon 129 allele. $\mathrm{PrP}^{\mathrm{Sc}}$ type 1 presents in $95 \%$ of the sporadic CJD patients who are MM homozygous, whereas $\mathrm{PrP}^{\mathrm{Sc}}$ type 2 was present in $86 \%$ of the patients who are either VV homozygous or MV heterozygous ${ }^{5,54}$ The idea that each polymorphism can facilitate two different ways of $\mathrm{PrP}^{\mathrm{C}} \rightarrow \mathrm{PrP}^{\mathrm{Sc}}$ interconversion is consistent with these observations. If our hypothesis is correct, we predict that $\mathrm{PrP}^{\mathrm{Sc}}$ type 1 , which correlates with the $\mathrm{M}$ genotype, arises from a $\operatorname{PrP}^{\mathrm{C}}$ $\rightarrow \mathrm{PrP}^{\mathrm{Sc}}$ that proceeds by a mechanism initiated by the breaking of the $\operatorname{PrP}^{\mathrm{C}}$ native $\beta$-sheet structure, while $\mathrm{PrP}^{\mathrm{Sc}}$ type 2 correlates with the $\mathrm{V}$ genotype and follows a $\operatorname{PrP}^{\mathrm{C}} \rightarrow \operatorname{PrP}^{\mathrm{Sc}}$ reaction through a native $\beta$-sheet elongation mechanism.

In the most simplistic projection of this model, the misfolding of a $\operatorname{PrP}^{\mathrm{C}}$ through $\beta$-sheet breaking should lead to a $\operatorname{PrP}^{\mathrm{Sc}}$ type 1 that catalyzes further $\beta$-sheet breaking. In this approach, is reasonable to consider that the recruiting of $\mathrm{PrP}^{\mathrm{Sc}}$ type 1 will be significantly affected if many of the $\operatorname{PrP}^{\mathrm{C}}$ genotypes that reacts are resistant to the $\beta$-sheet breaking. The same reasoning applies to the elongation mechanism and $\mathrm{PrP}^{\mathrm{Sc}}$ type 2. Here we are considering that the 129 allele is only tilting the thermodynamic balance to one or other reaction, thus an unfavorable crossing catalysis might still be possible. The observation that homozygosity is not a fully restrictive condition to prion disease might arise from this consideration.

Our hypothesis postulates the existence of a "fork" in the $\mathrm{PrP}^{\mathrm{C}} \rightarrow \mathrm{PrP}^{\mathrm{Sc}}$ reaction mechanism that can be affected by the polymorphism $129 \mathrm{via}$ its influence on the $\beta$-sheet structure stability. This idea of course does not clarify the full mechanism of $\mathrm{PrP}^{\mathrm{C}} \rightarrow \mathrm{PrP}^{\mathrm{Sc}}$ reaction and does not gives any direct insight in the $\operatorname{PrP}^{\mathrm{Sc}}$ aggregation process. Furthermore, besides the classification scheme of Parchi et al., other classification systems exist and more $\operatorname{PrP}^{\mathrm{Sc}}$ types have been observed, ${ }^{9,55}$ and the existence of several $\operatorname{PrP}^{\mathrm{Sc}}$ types was considered a potential cause. ${ }^{3}$ Therefore, it is reasonable to consider the possibility of other forks in the $\mathrm{PrP}^{\mathrm{C}} \rightarrow \operatorname{PrP}^{\mathrm{Sc}}$ reaction mechanism, ${ }^{56}$ other thermodynamic/kinetic determinants in the aggregation process $^{4}$ or even the action of other biomolecules in this process. Nevertheless, the results presented here might give a thermodynamic/kinetic explanation to the important influence of residue 129 in the different disease phenotypes and susceptibilities.

\section{Conclusion}

We have reported results of all-atom free-energy calculations assessing the stability of the native $\mathrm{N}$-terminal $\beta$-sheet of native human $\operatorname{PrP}^{\mathrm{C}}$ using the new method of replica-exchange on-the- fly-parameterization (RE-OTFP). We showed unprecedentedly high precision of the free-energy landscapes $\left(\ll 1 \mathrm{kcal} \mathrm{mol}^{-1}\right)$ computed using RE-OTFP and illustrated that RE-OTFP is vastly cheaper on a per-unit-error basis than any current collectivevariable biasing free-energy method. We compared free-energy landscapes for the four genotypes arising from the M129V/ D178N polymorphs of the human $\operatorname{PrP}^{\mathrm{C}}$, which were selected due to the singular importance of these two residues in determining variability of TSE phenotypes. Although all landscapes had the same ground state structure, which agrees with experiment for all polymorphs, we showed clear differences among these freeenergy landscapes in the form of new metastabilities. This led us to hypothesize that TSE strains might originate from differences in the energetic accessibility of metastable states that select for either elongation or breaking of the native $\beta$-sheet on pathways to distinct infectious $\operatorname{PrP}^{\mathrm{Sc}}$ conformations. The marginal effect of polymorphism 129 in the equilibrium configuration together with the FELs presented indicate that the study of excited $\operatorname{PrP}^{\mathrm{C}}$ configurations might lead to further insights in the prion phenotype phenomena. Finally, the precision of the FELs generating using RE-OTFP indicate that it might be the method of choice for characterizing thermodynamic stability differences of protein conformations arising from seemingly conservative mutations that do not introduce or destroy any specific interactions but nonetheless lead to distinct functional phenotypes.

\section{Acknowledgements}

Direct financial support from National Science Foundation (DMR-1207389) is gratefully acknowledged. Work reported here was run on hardware supported by Drexel's University Research Computing Facility and by the Extreme Science and Engineering Discovery Environment (XSEDE), ${ }^{57}$ which is supported by National Science Foundation grant number ACI-1053575.

\section{References}

1 S. B. Prusiner, Proc. Natl. Acad. Sci. U. S. A., 1998, 95, 1336313383.

2 R. Morales, K. Abid and C. Soto, Biochim. Biophys. Acta, Mol. Basis Dis., 2007, 1772, 681-691.

3 J. Collinge and A. R. Clarke, Science, 2007, 318, 930-936.

4 R. Morales, P. P. Hu, C. Duran-Aniotz, F. Moda, R. DiazEspinoza, B. Chen, J. Bravo-Alegria, N. Makarava, I. V. Baskakov and C. Soto, Sci. Rep., 2016, 6, 20526.

5 P. Gambetti, I. Cali, S. Notari, Q. Kong, W.-q. Zou and W. K. Surewicz, Acta Neuropathol., 2011, 121, 79-90.

6 P. Parchi, A. Giese, S. Capellari, P. Brown, W. SchulzSchaeffer, O. Windl, I. Zerr, H. Budka, N. Kopp, P. Piccardo, S. Poser, A. Rojiani, N. Streichemberger, J. Julien, C. Vital, B. Ghetti, P. Gambetti and H. Kretzschmar, Ann. Neurol., 1999, 46, 224-233.

7 P. Parchi, R. Strammiello, S. Notari, A. Giese, J. P. M. Langeveld, A. Ladogana, I. Zerr, F. Roncaroli, P. Cras, B. Ghetti, M. Pocchiari, H. Kretzschmar and S. Capellari, Acta Neuropathol., 2009, 118, 659-671. 
8 G. Puoti, A. Bizzi, G. Forloni, J. G. Safar, F. Tagliavini and P. Gambetti, Lancet Neurol., 2012, 11, 618-628.

9 J. D. F. Wadsworth and J. Collinge, Acta Neuropathol., 2011, 121, 69-77.

10 S. Mead, Science, 2003, 300, 640-643.

11 A. Aguzzi and A. M. Calella, Physiol. Rev., 2009, 89, 11051152.

12 M. Imran and S. Mahmood, Virol. J., 2011, 8, 559.

13 W. J. Huang, W. W. Chen and X. Zhang, Eur. Rev. Med. Pharmacol. Sci., 2015, 19, 4028-4034.

14 J. C. Watts, K. Giles, A. Serban, S. Patel, A. Oehler, S. Bhardwaj, S. Guan, M. D. Greicius, B. L. Miller, S. J. DeArmond, M. D. Geschwind and S. B. Prusiner, Ann. Neurol., 2015, 78, 540-553.

15 I. Zerr, K. Kallenberg, D. M. Summers, C. Romero, A. Taratuto, U. Heinemann, M. Breithaupt, D. Varges, B. Meissner, A. Ladogana, M. Schuur, S. Haik, S. J. Collins, G. H. Jansen, G. B. Stokin, J. Pimentel, E. Hewer, D. Collie, P. Smith, H. Roberts, J. P. Brandel, C. Van Duijn, M. Pocchiari, C. Begue, P. Cras, R. G. Will and P. SanchezJuan, Brain, 2009, 132, 2659-2668.

16 S. J. Collins, Brain, 2006, 129, 2278-2287.

17 P. Gambetti, Q. Kong, W. Zou, P. Parchi and S. G. Chen, Br. Med. Bull., 2003, 66, 213-239.

18 N. Nathanson, J. Wilesmith and C. Griot, Am. J. Epidemiol., 1997, 145, 959-969.

19 A. Maheshwari, M. Fischer, P. Gambetti, A. Parker, A. Ram, C. Soto, L. Concha-Marambio, Y. Cohen, E. D. Belay, R. A. Maddox, S. Mead, C. Goodman, J. S. Kass, L. B. Schonberger and H. M. Hussein, Emerging Infect. Dis., 2015, 21, 750-759.

20 S. Mead, S. P. Mahal, J. Beck, T. Campbell, M. Farrall, E. Fisher and J. Collinge, Am. J. Hum. Genet., 2001, 69, 1225-1235.

21 P. Gambetti, P. Parchi, R. B. Petersen, S. G. Chen and E. Lugaresi, Brain Pathol., 1995, 5, 43-51.

22 S. Prigent and H. Rezaei, Prion, 2011, 5, 69-75.

23 A. Barducci, R. Chelli, P. Procacci, V. Schettino, F. L. Gervasio and M. Parrinello, J. Am. Chem. Soc., 2006, 128, 2705-2710.

24 A. Barducci, G. Bussi and M. Parrinello, Phys. Rev. Lett., 2008, 100, 20603.

25 S. A. Paz and C. F. Abrams, J. Chem. Theory Comput., 2015, 11, 5024-5034.

26 L. Zheng, M. Chen and W. Yang, J. Chem. Phys., 2009, 130, 234105-234115.

27 D. A. Pearlman and P. A. Kollman, J. Chem. Phys., 1989, 91, 7831.

28 C. F. Abrams and G. Bussi, Entropy, 2014, 16, 163-199.

29 H. Zheng, R. K. Smith, Y.-W. Jun, C. Kisielowski, U. Dahmen and A. P. Alivisatos, Science, 2009, 324, 1309-1312.

30 L. Zheng and W. Yang, J. Chem. Theory Comput., 2012, 8, 810823.

31 L. Zheng, M. Chen and W. Yang, Proc. Natl. Acad. Sci. U. S. A., 2008, 105, 20227-20232.

32 C. Lu, X. Li, D. Wu, L. Zheng and W. Yang, J. Chem. Theory Comput., 2016, 12, 41-52.

33 U. H. Hansmann, Chem. Phys. Lett., 1997, 281, 140-150.
34 D. J. Earl and M. W. Deem, Phys. Chem. Chem. Phys., 2005, 7, 3910-3916.

35 W. Zhang, C. Wu and Y. Duan, J. Chem. Phys., 2005, 123, 1-9. 36 J. F. Dama, M. Parrinello and G. A. Voth, Phys. Rev. Lett., 2014, 112, 240602.

37 M. Deighan, M. Bonomi and J. Pfaendtner, J. Chem. Theory Comput., 2012, 8, 2189-2192.

38 G. Bussi, F. L. Gervasio, A. Laio and M. Parrinello, J. Am. Chem. Soc., 2006, 128, 13435-13441.

39 E. Darve and A. Pohorille, J. Chem. Phys., 2001, 115, 9169.

40 E. Darve, D. Rodríguez-Gómez and A. Pohorille, J. Chem. Phys., 2008, 128, 144120.

41 C. F. Abrams and E. Vanden-Eijnden, Chem. Phys. Lett., 2012, 547, 114-119.

42 L. Maragliano, A. Fischer, E. Vanden-Eijnden and G. Ciccotti, J. Chem. Phys., 2006, 125, 24106.

43 C. F. Abrams and E. Vanden-Eijnden, Proc. Natl. Acad. Sci. U. S. A., 2010, 107, 4961-4966.

44 Y. Sugita and Y. Okamoto, Chem. Phys. Lett., 1999, 314, 141-151. 45 A. Kone and D. A. Kofke, J. Chem. Phys., 2005, 122, 1-2.

46 N. Rathore, M. Chopra and J. J. de Pablo, J. Chem. Phys., 2005, 122, 024111.

47 J. C. Phillips, R. Braun, W. Wang, J. Gumbart, E. Tajkhorshid, E. Villa, C. Chipot, R. D. Skeel, L. Kalé and K. Schulten, J. Comput. Chem., 2005, 26, 1781-1802.

48 A. D. MacKerell Jr, D. Bashford, M. Bellott, R. L. Dunbrack Jr, J. D. Evanseck, M. J. Field, S. Fischer, J. Gao, H. Guo, S. Ha, D. Joseph-McCarthy, L. Kuchnir, K. Kuczera, F. T. K. Lau, C. Mattos, S. Michnick, T. Ngo, D. T. Nguyen, B. Prodhom, W. E. Reiher III, B. Roux, M. Schlenkrich, J. C. Smith, R. Stote, J. Straub, M. Watanabe, J. Wiorkiewicz-Kuczera, D. Yin and M. Karplus, J. Phys. Chem. B, 1998, 102, 3586-3616. 49 A. D. Mackerell, M. Feig and C. L. Brooks, J. Comput. Chem., 2004, 25, 1400-1415.

50 L. Calzolai and R. Zahn, J. Biol. Chem., 2003, 278, 35592-35596. 51 S. Lee, L. Antony, R. Hartmann, K. J. Knaus, K. Surewicz, W. K. Surewicz and V. C. Yee, EMBO J., 2010, 29, 251-262.

52 L. L. P. Hosszu, G. S. Jackson, C. R. Trevitt, S. Jones, M. Batchelor, D. Bhelt, K. Prodromidou, A. R. Clarke, J. P. Waltho and J. Collinge, J. Biol. Chem., 2004, 279, 28515-28521.

53 L. L. P. Hosszu, M. A. Wells, G. S. Jackson, S. Jones, M. Batchelor, A. R. Clarke, C. J. Craven, J. P. Waltho and J. Collinge, Biochemistry, 2005, 44, 16649-16657.

54 P. Parchi, R. Castellani, S. Capellari, B. Ghetti, K. Young, S. G. Chen, M. Farlow, D. W. Dickson, A. A. F. Sima, J. Q. Trojanowski, R. B. Petersen and P. Gambetti, Ann. Neurol., 1996, 39, 767-778.

55 A. F. Hill, S. Joiner, J. D. F. Wadsworth, K. C. L. Sidle, J. E. Bell, H. Budka, J. W. Ironside and J. Collinge, Brain, 2003, 126, 1333-1346.

56 J. Garrec, I. Tavernelli and U. Rothlisberger, PLoS Comput. Biol., 2013, 9, e1003057.

57 J. Towns, T. Cockerill, M. Dahan, I. Foster, K. Gaither, A. Grimshaw, V. Hazlewood, S. Lathrop, D. Lifka, G. D. Peterson, R. Roskies, J. R. Scott and N. WilkensDiehr, Comput. Sci. Eng., 2014, 16, 62-74. 